\title{
Capital Cost-Aware Design and Partial Shading-Aware Architecture Optimization of a Reconfigurable Photovoltaic System
}

\author{
Yanzhi Wang, Xue Lin, and Massoud Pedram \\ University of Southern California \\ Los Angeles, CA, USA 90089 \\ \{yanzhiwa, xuelin, pedram\}@usc.edu
}

\author{
Jaemin Kim and Naehyuck Chang \\ Seoul National University \\ Seoul, Korea 151-744 \\ \{jmkim, naehyuck\}@elpl.snu.ac.kr
}

\begin{abstract}
Photovoltaic (PV) systems are often subject to partial shading that significantly degrades the output power of the whole systems. Reconfiguration methods have been proposed to adaptively change the PV panel configuration according to the current partial shading pattern. The reconfigurable $P V$ panel architecture integrates every PV cell with three programmable switches to facilitate the PV panel reconfiguration. The additional switches, however, increase the capital cost of the PV system. In this paper, we group a number of $P V$ cells into a PV macro-cell, and the PV panel reconfiguration only changes the connections between adjacent PV macro-cells. The size and internal structure (i.e., the series-parallel connection of PV cells) of all PV macro-cells are the same and will not be changed after PV system installation in the field. Determining the optimal size of the PV macro-cell is the result of a trade-off between the decreased PV system capital cost and enhanced PV system performance. A larger PV macro-cell reduces the cost overhead whereas a smaller PV macro-cell achieves better performance. In this paper, we set out to calculate the optimal size of the PV macro-cells such that the maximum system performance can be achieved subject to an overall system cost limitation. This "design" problem is solved using an efficient search algorithm. In addition, we provide for in-field reconfigurability of the PV panel by enabling formation of seriesconnected groups of parallel-connected macro-cells. We ensure maximum output power for the PV system in response to any incurring partial shading pattern. This "architecture optimization" problem is solved using dynamic programming.
\end{abstract}

\section{INTRODUCTION}

Renewable energy technologies are vigorously promoted to achieve sustainability of the economic and social development of humanity. Since solar energy is abundant and easily accessible, photovoltaic (PV) energy generation techniques cause great interest and attention. The output power of PV systems is directly dependent on the solar irradiance level, which is changing frequently according to the time of day and weather conditions. This is a major drawback of PV systems. Standalone PV systems are equipped with electrical energy storage elements to store the excess output electrical energy under high levels of solar irradiance and compensate the insufficient output electrical energy under low levels of solar irradiance [1].

PV panels exhibit highly non-linear output current-voltage (I-V) characteristics that change with solar irradiance. Therefore, a charger is needed between the PV panel and the load device in a PV system to provide a controllable current for the load device. Usually, maximum power point tracking (MPPT) techniques are integrated into the charger to extract the maximum power from the PV panel under any solar irradiance levels [2]. Recently, the maximum power transfer tracking (MPTT) method, which accounts for changes in the charger efficiency, can be more effective than the MPPT methods [3].

A conventional PV panel consists of $m \times n$ PV cells connected in a series-parallel manner, where $m P V$ cell groups are connected in series and each PV cell group has $n$ PV cells connected in parallel, so as to achieve desirable output voltage and current. Solar irradiance received by PV cells in the PV panel may be different when a part of the PV panel is shaded. Such a phenomenon is known as partial

This work is supported in part by a grant from the U.S. National Science Foundation, and by a grant from the BK21 Project and the NRF of Korea funded by the MEST (No. 2012054193). shading. Partial shading is commonly caused by moving clouds and shadows of nearby obstacles on a PV. Partial shading not only reduces the maximum output power of the shaded PV cell itself, but also makes the non-shaded PV cells that are in series with the shaded one deviate from their maximum power points (MPPs ${ }^{1}$ ). This makes the maximum output power of a PV panel much lower than the sum of MPP output power values of all the PV cells in the PV panel.

PV panel reconfiguration methods can maintain the output power of the PV system under partial shading by adaptively changing the PV panel configuration, i.e., the connection topology of PV cells. A PV panel reconfiguration method is proposed in [4] that significantly improves the output power of the PV system under partial shading. A conventional fixed $m \times n$ PV panel structure is replaced with a reconfigurable PV panel structure, where each PV cell is integrated with three switches. The PV panel configuration can be changed by controlling the on/off states of the switches. A reconfiguration control algorithm is proposed to find the near-optimal configuration of the PV panel according to the current partial shading pattern such that the output power of the PV system is always maximized.

However, the additional programmable switches used for PV panel reconfiguration increase the capital cost of the whole PV system. In [4], the basic unit of the PV panel reconfiguration is one single PV cell, i.e., PV panel reconfiguration changes the connection of the PV cells. In this paper, we use the PV macro-cell as the basic unit of the PV panel reconfiguration method. The PV macro-cell consists of a number of PV cells connected in series and parallel. The PV panel reconfiguration changes the connection of PV macro-cells while the size and structure of the PV macro-cell are determined at the system design stage. The size of the PV macro-cell is essentially trade-off between the lower additional capital cost and performance enhancement. Basically, a larger PV macro-cell reduces the cost of the reconfigurable PV panel architecture since fewer switches are required for reconfiguration, while a smaller PV macro-cell achieves better flexibility and thus higher performance against partial shading. We provide an algorithm to decide the optimal size of the PV macrocell for the PV system design, thereby achieving a balance between the capital cost and performance. Experimental results demonstrate $60 \%$ enhancement in PV system output power with a negligible capital cost increase of $3.7 \%$ by selecting the proper macro-cell size.

\section{COMPONENT MODELS}

\section{A. PV Cell Model and Characterization}

Every PV panel consists of multiple PV cells. Let $V_{p v c}$ and $I_{p v c}$ denote the output voltage and current of a PV cell, respectively. The relationship between $I_{p v c}$ and $V_{p v c}$ (i.e., the I-V characteristics) with a given solar irradiance $G$ is provided in Eqn. (1) of [4]. Figure 1 illustrates the PV cell I-V and P-V characteristics under different solar irradiance levels, where $G_{S T C}$ stands for the irradiance (1000 $\mathrm{W} / \mathrm{m}^{2}$ ) at standard test condition. One can observe that the PV cell exhibits a non-linear output current and voltage relationship. There is a MPP under any solar irradiance level, where the output power of the PV cell is maximized. MPPs are labeled by red dots in Figure 1.

${ }^{1}$ On the I-V output characteristics of a PV cell, there is a point $(V, I)$ where output power is maximized. This point is the MPP of the PV cell. 

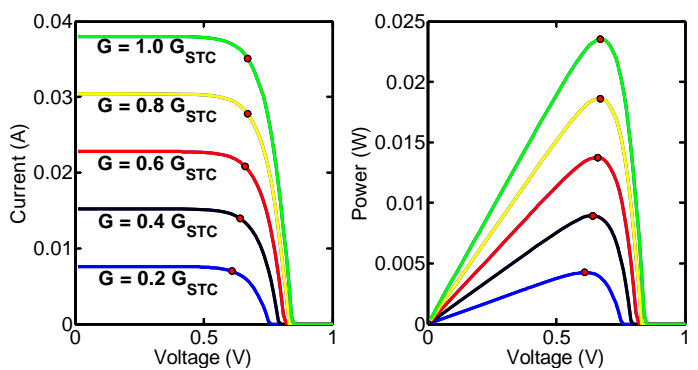

Figure 1: I-V and P-V output characteristics of a PV cell.

\section{B. Charger Model}

We use a charger between the PV panel and the energy storage device. The charger model is introduced in [5]. $V_{\text {in }}, I_{\text {in }}, V_{\text {out }}$, and $I_{\text {out }}$ denote the input voltage, input current, output voltage and output current of the charger, respectively. The input ports of the charger are connected to the PV panel, and the output ports are connected to the load. The system controller adjusts the charger output current, thereby maintaining the operating point of the PV panel. The power consumption of the charger $P_{c o n v}$ is a function of $V_{i n}, I_{\text {in }}$, and $V_{\text {out }}$ [5]. Based on this model, we can calculate the charger output current as a function of $V_{\text {in }}, I_{\text {in }}$, and $V_{\text {out }}: I_{\text {out }}=C h g_{-}$Out_I $\left(V_{\text {in }}, I_{\text {in }}, V_{\text {out }}\right)$.

\section{RECONFIGURABLE PV PANEL ARCHITECTURE}

The conventional PV panel (with fixed configuration) is replaced with the reconfigurable one for the PV system to combat partial shading. The physical locations of the PV cells/macro-cells are fixed after the PV system installation. The PV panel reconfiguration only changes the electrical connection of PV cells/macro-cells. The reconfigurable PV panel architecture is shown in Figure 2, which represents the electrical connection of $N$ PV macro-cells instead of the actual physical locations of PV macro-cells. Each PV macro-cell except for the last one is integrated with three switches i.e., a top Pswitch $S_{P T, i}$, a bottom P-switch $S_{P B, i}$, and a S-switch $S_{S, i}$. In Figure 2, the PV macro-cell has a size of $2 \times 2$.

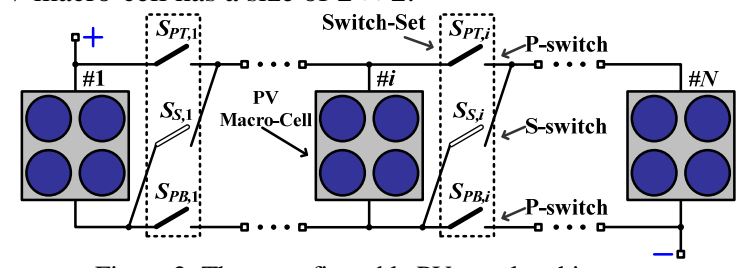

Figure 2: The reconfigurable PV panel architecture.

Each switch in this architecture consists of one MOSFET gate driver and one or multiple pairs of n-type MOSFETs. The two MOSFETs in one MOSFET pair are connected back to back. Figure 3 shows the detailed circuitry of a switch. MOSFET pairs can be connected in parallel in case that the PV panel produces higher current than the maximum drain current of a MOSFET. The minimum required number of MOSFETs for a switch is calculated by

$$
N_{\text {minMos }}=2 \times\left\lceil\frac{N \cdot p \cdot \operatorname{MAX}\left(I_{p v c}\right)}{I_{D}}\right\rceil,
$$

where $p$ is the number of PV cells connected in parallel in a macrocell, and $I_{D}$ is the maximum drain current of a MOSFET. Therefore, the required number of MOSFETs per $\mathrm{PV}$ macro-cell is $3 \cdot N_{\text {minmos }}$.

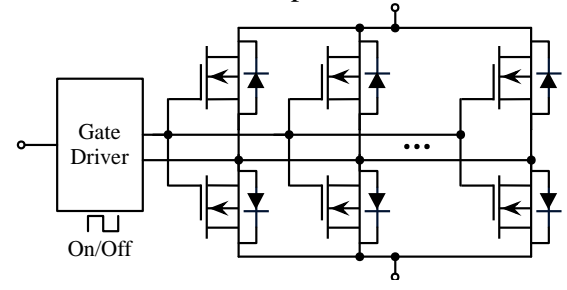

Figure 3: The switch circuitry.
The PV panel reconfiguration can be conducted by controlling the on/off states of these switches. The two P-switches of a PV macrocell are always in the same state, and its S-switch must be in the opposite state. The P-switches connect PV macro-cells in parallel to form a $P V$ group, and the $\mathrm{S}$-switches connect the PV groups in series.
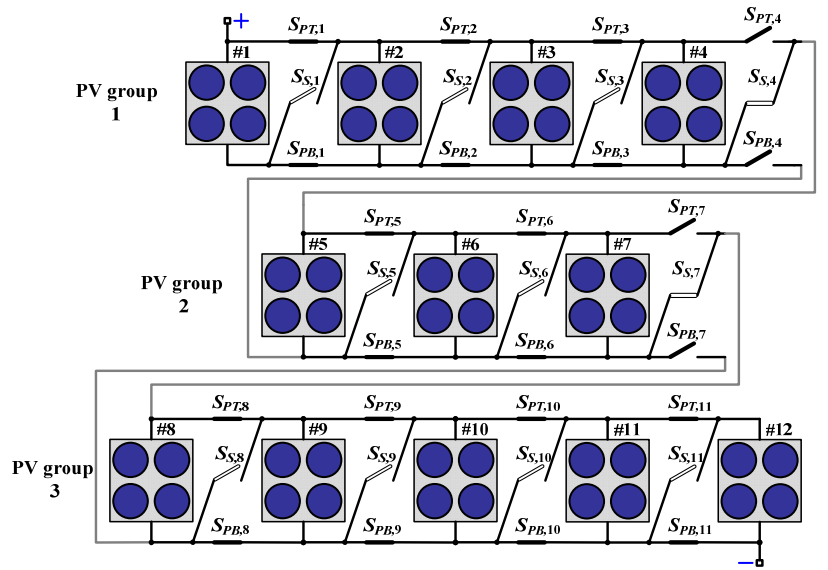

Figure 4: An example of PV panel reconfiguration.

Figure 4 is an example of PV panel reconfiguration. Figure 4 presents the electrical connection of PV macro-cells instead of their physical locations. The first four PV macro-cells are connected in parallel to form PV group 1; the next three PV macro-cells form PV group 2; and the last five PV macro-cells form PV group 3. These three PV groups are series-connected by the S-switches of the fourth and the seventh PV macro-cells.

A reconfigurable $\mathrm{PV}$ panel consisting of $N$ PV macro-cells includes an arbitrary number (less than or equal to $N$ ) of PV groups. The number of parallel-connected PV macro-cells $r_{j}(>0)$ in the $j$-th PV group should satisfy

$$
\sum_{j=1}^{g} r_{j}=N
$$

where $g$ is the number of PV groups. We denote such a configuration by $\mathcal{C}\left(g ; r_{1}, r_{2}, \cdots, r_{g}\right)$. This configuration can be viewed as a partition of the PV macro-cell index set $\boldsymbol{A}=\{1,2,3, \cdots, N\}$, where the elements in $\boldsymbol{A}$ denote the indices of PV macro-cells in the PV panel. The partition is denoted by subsets $\boldsymbol{B}_{1}, \boldsymbol{B}_{2}, \cdots$, and $\boldsymbol{B}_{g}$ of $\boldsymbol{A}$, which correspond to the $g \mathrm{PV}$ groups consisting of $r_{1}, r_{2}, \cdots$, and $r_{g} \mathrm{PV}$ macro-cells, respectively. The subsets $\boldsymbol{B}_{1}, \boldsymbol{B}_{2}, \cdots$, and $\boldsymbol{B}_{g}$ satisfy

$$
\bigcup_{j=1}^{g} B_{j}=A,
$$

and

$$
\boldsymbol{B}_{j} \cap \boldsymbol{B}_{k}=\emptyset \text {, for } \forall j, k \in\{1,2, \cdots, g\} \text { and } j \neq k \text {. }
$$

The indices of PV macro-cells in PV group $j$ must be smaller than the indices of PV macro-cells in PV group $k$ for any $1 \leq j<k \leq g$ due to the structural characteristics of the reconfigurable PV panel architecture, i.e., $i_{1}<i_{2}$ for $\forall i_{1} \in \boldsymbol{B}_{j}$ and $\forall i_{2} \in \boldsymbol{B}_{k}$ satisfying $1 \leq j<k \leq g$. A partitioning satisfying the above properties is called an alphabetical partitioning.

The PV cells in a PV panel are usually physically placed into an $m \times n$ array, where there are $m$ rows and $n$ columns of PV cells. This is the physical (not electrical) layout of a PV panel. The PV macrocell has a size of $s \times p$, i.e., $s$ PV cells connected in series and $p$ PV cells connected in parallel to form a PV macro-cell. $s$ and $p$ must be factors of $m$ and $n$, respectively. The PV macro-cells (from 1 to $N$ ) are physically placed in a zigzag manner in the PV panel. In this way, the total length of wires connecting the PV macro-cells can be minimized. The PV panel reconfiguration method only changes the electrical connection of the PV macro-cells, whereas the electrical connection of PV cells within a macro-cell is fixed after PV system installation in the field. 


\section{OPTIMIZATION AlgORITHM}

We first discuss the macro-cell based PV panel reconfiguration problem targeting at maximizing the output power of the PV system against partial shading. In this problem we assume that the size of the PV macro-cell is given. Secondly, we discuss the PV system design optimization problem to determine the optimal macro-cell size.

\section{A. PV Panel Reconfiguration Algorithm}

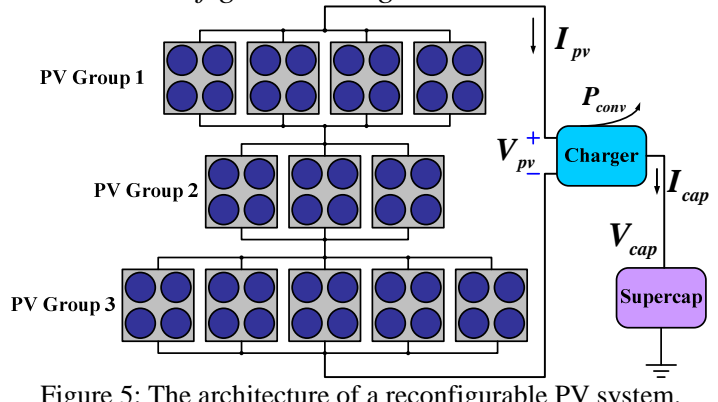

Figure 5: The architecture of a reconfigurable PV system.

Figure 5 shows a reconfigurable PV system comprised of a reconfigurable PV panel, a charger and a supercapacitor as the energy storage. The PV cells in the PV panel are placed into an $m \times n \mathrm{PV}$ cell array (not shown in Figure 5). The PV macro-cell has a size of $s \times p$. Then the total number of PV macro-cells is $N=(m \times$ $n) /(s \times p)$. The output voltage and current of the PV panel are $V_{p v}$ and $I_{p v}$, respectively. The power consumption of the charger is $P_{c o n v}$. The terminal voltage of the supercapacitor is $V_{c a p}$, and the charging current of the supercapacitor is $I_{\text {cap }}$. We have

$$
V_{p v} \cdot I_{p v}=P_{c o n v}+V_{c a p} \cdot I_{c a p} .
$$

We assume that the PV reconfiguration controller has no access to the solar irradiance on every PV cell for the realistic concern. Instead, it has only an estimate $\widehat{G}_{i}^{m}$ of the solar irradiance on each $i$-th PV macro-cell. This estimate causes certain performance degradation since PV cells in a macro-cell may receive different irradiances. The formal statement of the PV panel reconfiguration problem is:

PV Panel Reconfiguration Problem Statement: Referring to Figure 5, given the solar irradiance estimate $\widehat{G}_{i}^{m}$ on the $i$-th $(1 \leq i \leq$ $N) \mathrm{PV}$ macro-cell and the supercapacitor terminal voltage $V_{\text {cap }}$, find the optimal PV panel configuration $\mathcal{C}^{\text {opt }}$ and the optimal PV panel operating point $\left(V_{p v}^{o p t}, I_{p v}^{o p t}\right)$, such that $I_{c a p}$ is maximized.

We propose the near-optimal PV panel reconfiguration algorithm consisting of a kernel algorithm and an outer loop. The kernel algorithm finds the optimal $r_{1}, r_{2}, \cdots, r_{g}$ with a given $g$ such that the PV panel MPP power is maximized. The PV panel reconfiguration method only changes the connection of PV macro-cells while the size and structure of a PV macro-cell are fixed. The kernel algorithm is based on dynamic programming with polynomial time complexity. The outer loop determines the optimal $g$ value.

Similar to [4], the kernel algorithm also relies on the observation that the MPP voltages of a PV cell under different solar irradiance levels are very close to each other, but the corresponding MPP currents vary significantly. Let $V_{p v c}^{M P P}$ denote the solar irradianceindependent MPP voltage of a PV cell, and let $I_{p v c}^{M P P}(G)$ denote its MPP current as a function of the solar irradiance $G$. The kernel algorithm essentially treats the PV macro-cell as a super PV cell, whose length is $s$ times of that of the original PV cell and width is $p$ times of that of the original PV cell. The kernel algorithm assumes that each $i$-th PV macro-cell receives uniform solar irradiance $\hat{G}_{i}^{m}$. It estimates the MPP voltage and current of each $i$-th PV macro-cell as $s \cdot V_{p v c}^{M P P}$ and $\hat{I}_{p v m c, i}^{M P P}=p \cdot I_{p v c}^{M P P}\left(\hat{G}_{i}^{m}\right)$, respectively. The kernel algorithm maximizes the estimated PV panel MPP power given by

$$
g \cdot s \cdot V_{p v c}^{M P P} \cdot \min _{j} \sum_{i \in B_{j}} p \cdot I_{p v c}^{M P P}\left(\hat{G}_{i}^{m}\right) .
$$

Or equivalently, it maximizes $\min _{j} \sum_{i \in \boldsymbol{B}_{j}} p \cdot I_{p v c}^{M P P}\left(\hat{G}_{i}^{m}\right)$. Optimal substructure property similar to that in [4] also applies to this problem. Hence, we apply dynamic programming method as the basis of the kernel algorithm to solve this problem. Details of the kernel algorithm are provided in Algorithm 2.

In the outer loop, we first run Algorithm 2 to find an optimal configuration $\mathcal{C}^{\text {opt }}(g)$ for each $g$ value. After that, we calculate the estimated $I_{c a p}$ under such configuration using the function Chg_Out_I $\left(V_{p v}, I_{p v}, V_{c a p}\right)$. In the calculation of the estimated $I_{c a p}$, we estimate the PV panel output voltage and current by $g \cdot s \cdot V_{p v c}^{M P P}$ and $\min _{j} \sum_{i \in \boldsymbol{B}_{j}} p \cdot I_{p v c}^{M P P}\left(\hat{G}_{i}^{m}\right)$, respectively. We find the optimal $g$ value that maximizes the estimated $I_{c a p}$, and the corresponding configuration $\mathcal{C}^{o p t}$. Details are provided in Algorithm 1.

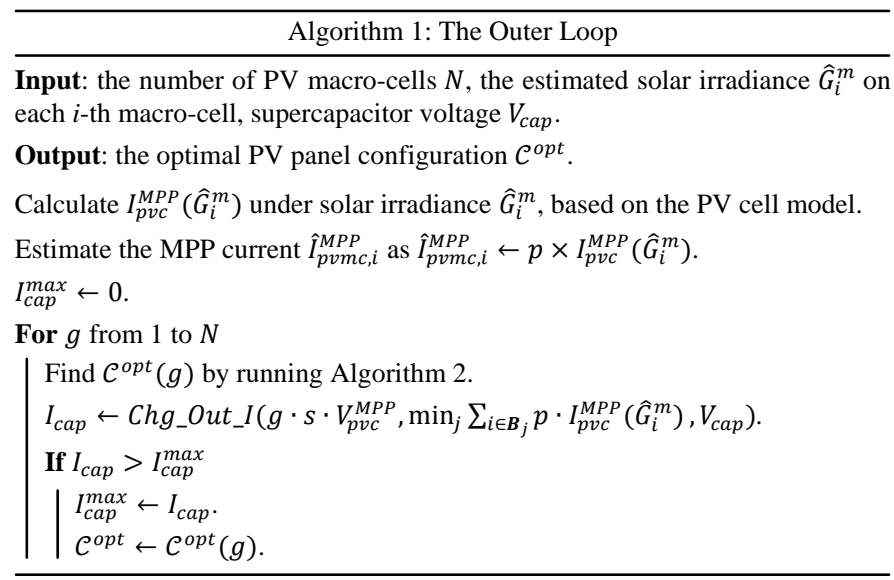

Algorithm 2: The Kernel Algorithm

Input: the number of PV macro-cells $N$, the number of PV groups $g$, the estimated MPP current of each $i$-th PV macro-cell $\hat{I}_{p v m c, i}^{M P P}$.

Output: the optimal PV panel configuration $\mathcal{C}^{\text {opt }}(g)$.

Maintain two $N \times g$ matrixes Min_Sum_Opt and Last_Par.

Initialize in_Sum_Opt $\left(l_{1}, 1\right) \leftarrow \sum_{1 \leq i \leq l_{1}} \hat{I}_{p v m c, i}^{M P P}$, Last_Par $\left(l_{1}, 1\right) \leftarrow 0$.

For $l_{2}$ from 2 to $g$

For $l_{1}$ from $l_{2}$ to $N$

Min_Sum_Opt $\left(l_{1}, l_{2}\right) \leftarrow$

$\max _{l_{2}-1 \leq l<l_{1}} \min \left\{\right.$ Min_Sum_Opt $\left.\left(l, l_{2}-1\right), \sum_{l<i \leq l_{1}} \hat{I}_{p v m c, i}^{M P P}\right\}$.

Last_Par $\left(l_{1}, l_{2}\right) \leftarrow$

$\arg \max _{l_{2}-1 \leq l<l_{1}} \min \left\{\right.$ Min_Sum_Opt $\left.\left(l, l_{2}-1\right), \sum_{l<i \leq l_{1}} \hat{I}_{p v m c, i}^{M P P}\right\}$.

Trace back using Last_Par to find the optimal configuration $\mathcal{C}^{\text {opt }}(g)$.

\section{B. PV System Design Optimization}

The PV system design optimization problem aims to find the optimal size (i.e., the optimal $s$ and $p$ ) of the PV macro-cell considering the trade-off between lower PV system capital cost and higher performance. When the PV macro-cell size is smaller, the PV system capital cost becomes higher due to the increased number of integrated switches. On the other hand, the PV system achieves higher performance in combating the partial shading effect when the macro-cell size is smaller because of the following two reasons: (i) The PV panel with a smaller macro-cell size has higher flexibility in reconfiguration against partial shading. (ii) The use of a single solar irradiance level for each PV macro-cell in the PV reconfiguration algorithm causes certain performance degradation.

The PV system design optimization is performed at the system design stage. We formulate the optimization problem as follows:

PV System Design Optimization Problem 1: Given a PV panel with fixed area and fixed physical layout of $m \times n$ PV cells, find the optimal $s$ and $p$, such that the PV system performance (output 
power) is maximized and the PV system capital cost is within a certain limit. $s$ and $p$ should be factors of $m$ and $n$, respectively.

The proposed PV system design optimization algorithm is provided in Algorithm 3. We utilize the following observation to reduce the search space to a Pareto-optimal set of $s$ and $p$ values.

Observation I: Consider two PV panels with the same area and the same physical layout of PV cells, but with different macro-cell sizes $(s, p)$ and $\left(s^{\prime}, p^{\prime}\right)$, respectively, where $s \leq s^{\prime}$ and $p \leq p^{\prime}$. The performance of the former PV panel is no less than that of the latter.

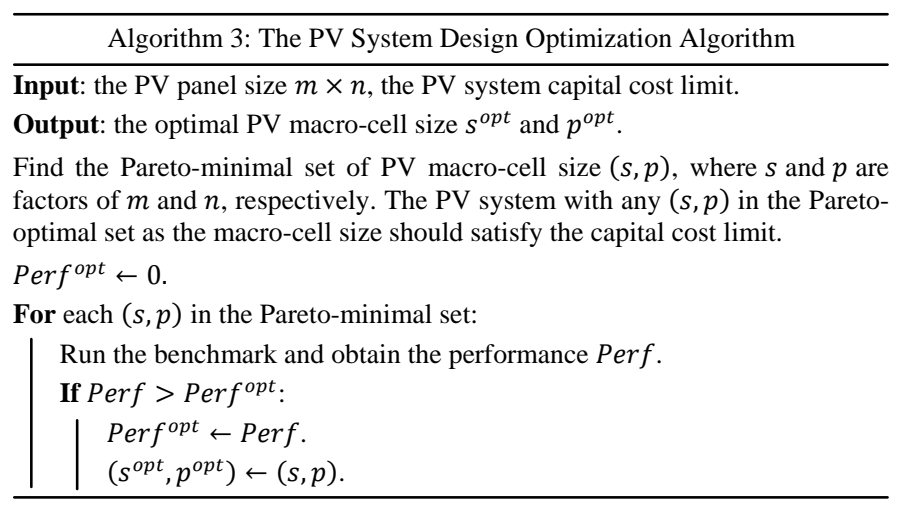

Similarly, we formulate PV System Design Optimization Problem $\mathbf{2}$ as minimizing the PV system capital cost with a performance degradation constraint. We evaluate the performance degradation with respect to the PV panel with macro-cell size $1 \times 1$. We exploit binary search based algorithm to find the optimal $s$ and $p$ values, making use of the observation that a small macro-cell size results in a higher PV system performance against partial shading.

\section{EXPERIMENTAL RESULTS}

We investigate the macro-cell size as a trade-off between the decreased capital cost and enhanced performance. We consider a $12 \times 12$ PV panel with 36 possible sizes of macro-cell. We exploit real-world examples of the PV cells, switches and switch drivers, with prices and specifications shown in Table 1 . We carefully select commercial PV cells in the price range of $\sim \$ 1, \sim \$ 5, \sim \$ 20$. We name them low-price, medium-price, and high-price PV cells, respectively.

Table 1: Characteristics of PV cells, MOSFETs and gate driver

\begin{tabular}{|c|c|c|c|}
\hline PV Cell & $I_{s c}$ & $P_{m p p}$ & Price \\
\hline SZGD6030 & $302 \mathrm{~mA}$ & $134.4 \mathrm{~mW}$ & $\$ 1.2$ \\
\hline SZGD10040-10 & $115 \mathrm{~mA}$ & $575 \mathrm{~mW}$ & $\$ 5.5$ \\
\hline SZGD196156-10 & $720 \mathrm{~mA}$ & $3250 \mathrm{~mW}$ & $\$ 21.9$ \\
\hline MOSFET & $V_{D S}$ & $I_{D}$ & Price \\
\hline DMG4406LSS-13 & $30 \mathrm{~V}$ & $10.3 \mathrm{~A}$ & $\$ 0.09$ \\
\hline Gate Driver & $V_{\max }$ & $T_{o n}$ & Price \\
\hline MAX15054AUT+T-ND & $65 \mathrm{~V}$ & $11 \mathrm{~ns}$ & $\$ 0.70$ \\
\hline
\end{tabular}

We effectively evaluate the performance of reconfigurable PV panels using PV panel partial shading benchmarks. The proposed benchmarks consist of (i) extreme-case partial shading patterns as those in [4] for testing the robustness of the PV panel reconfiguration architecture, (ii) random shading over each PV cell to mimic the effect of dusts on the PV panel, and (iii) block-based partial shading on the PV panel to mimic the effect of clouds.

Figure 6 shows the trade-off between decreased capital cost and enhanced performance of a PV panel consisting of medium-price PV cells when the PV macro-cell size changes. The $\mathrm{x}$-axis of Figure 6 is the percentage of performance enhancement over the same size PV panel without any reconfiguration method. The y-axis of Figure 6 is the percentage of capital cost decrease with respect to the same size PV panel with macro-cell size of $1 \times 1$. All the Pareto-optimal sizes of PV macro-cell are marked in Figure 6.

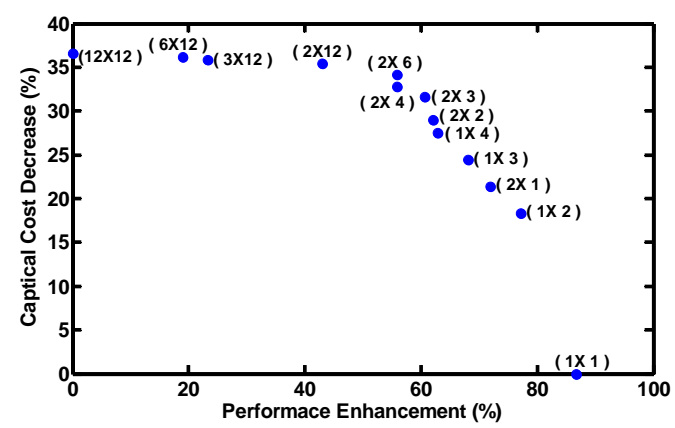

Figure 6: Pareto-optimal macro-cell sizes and the corresponding PV system performance and capital cost.

Table 2 provides experimental results on the PV system design optimization problem 2. It provides the minimal amounts of capital cost increase when the PV system performance is $20 \%, 40 \%, 60 \%$, and $80 \%$ higher than the PV system without any reconfiguration method. According to Table 2, the high-price PV cell-based panel achieves more than $60 \%$ performance improvement with a negligible capital cost increase of $3.7 \%$, compared with the PV panel without reconfiguration ability. Hence, it is more beneficial in investing on the reconfiguration architecture than simply increasing the area of the PV panel. On the other hand, the low-price PV cell-based panel encounters $354 \%$ capital cost increase in order to enhance its output power by $80 \%$, which is certainly not as cost-effective as increasing the area of the PV panel by $80 \%$ if allowable. However, investing on the reconfiguration architecture will be cost-effective even for lowprice PV cell-based panels if we set the macro-cell size relatively larger. For example, such PV panel achieves more than $40 \%$ performance gain with only $11 \%$ increase in capital cost. It is beneficial to invest on the reconfiguration architecture in this case.

Table 2: Minimal cost increase with performance guarantee

\begin{tabular}{|c|c|c|c|c|}
\hline Performance gain & $>20 \%$ & $>40 \%$ & $>60 \%$ & $>80 \%$ \\
\hline Low-price cell-based & $7.4 \%$ & $11 \%$ & $44 \%$ & $354 \%$ \\
\hline Medium-price cell-based & $1.3 \%$ & $2.0 \%$ & $8.0 \%$ & $58 \%$ \\
\hline High-price cell-based & $0.23 \%$ & $0.90 \%$ & $3.7 \%$ & $32 \%$ \\
\hline
\end{tabular}

\section{CONCLUSION}

Some PV panel reconfiguration methods have been proposed recently to adaptively change the PV panel configuration in order to combat the partial shading effect. The additional switches increase the capital cost of the PV system. In this paper, we propose a PV panel reconfiguration architecture and method based on the concept of PV macro-cell. A PV macro-cell consists of a group of PV cells, and the PV panel reconfiguration method only changes the connections between adjacent PV macro-cells while the size and structure of PV macro-cell are fixed during system operation. The size of a PV macro-cell is a trade-off between the decreased PV system capital cost and enhanced performance. We investigate the optimal size of the PV macro-cell such that the optimal performance can be guaranteed within the capital cost limitation, or vice versa.

\section{REFERENCES}

[1] J. Baker and A. Collinson, "Electrical energy storage at the turn of the millennium," Power Engineering Journal, 1999.

[2] F. Liu, S. Duan, F. Liu, B. Liu, and Y. Kang, "A variable step size INC MPPT method for PV systems," IEEE T. on Industrial Electronics, 2008.

[3] Y. Kim, N. Chang, Y. Wang, and M. Pedram, "Maximum power transfer tracking for a photovoltaic-supercapacitor energy system," ISLPED, Aug. 2010.

[4] X. Lin, Y. Wang, S. Yue, D. Shin, N. Chang, and M. Pedram, "Nearoptimal, dynamic module reconfiguration in a photovoltaic system to combat partial shading effects,” DAC, Jun. 2012.

[5] Y. Wang, Y. Kim, Q. Xie, N. Chang, and M. Pedram, "Charge migration efficiency optimization in hybrid electrical energy storage (HEES) systems," ISLPED, Aug. 2011. 